\title{
Associated Credit Risk Contagion with Incubatory Period: A Network-Based Perspective
}

\author{
Kai Xu $\left(\mathbb{D},{ }^{1}\right.$ Jianming Mo, ${ }^{2}$ Qian Qian $\left(\mathbb{D},{ }^{3}\right.$ Fengying Zhang, ${ }^{4}$ Xiaofeng Xie $\left(\mathbb{D},{ }^{5}\right.$ \\ and Zongfang Zhou ${ }^{6}$ \\ ${ }^{1}$ Business School, Chengdu University, No. 2025, Chengluo Avenue, Longquanyi District, Chengdu 610106, China \\ ${ }^{2}$ Chinese Finance Research Institute, Southwestern University of Finance and Economics, No. 55, Guanghua Village Road, \\ Wenjiang District, Chengdu 610074, China \\ ${ }^{3}$ School of Business, Sichuan Normal University, No. 5, Jinan Road, Jinjiang District, Chengdu 610101, China \\ ${ }^{4}$ West China School of Nursing, Sichuan University, No. 37, Guoxuexiang, Wuhou District, Chengdu 610041, China \\ ${ }^{5}$ West China School of Nursing/West China Hospital, Sichuan University, No. 37, Guoxuexiang, Wuhou District, \\ Chengdu 610041, China \\ ${ }^{6}$ School of Management and Economics, University of Electronic Science and Technology of China, No. 2006, Xiyuan Avenue, \\ High-Tech District, Chengdu 611731, China
}

Correspondence should be addressed to Kai Xu; xukai@cdu.edu.cn

Received 9 January 2020; Revised 20 June 2020; Accepted 17 July 2020; Published 17 August 2020

Academic Editor: Dehua Shen

Copyright ( $) 2020 \mathrm{Kai}$ Xu et al. This is an open access article distributed under the Creative Commons Attribution License, which permits unrestricted use, distribution, and reproduction in any medium, provided the original work is properly cited.

\begin{abstract}
Associated credit risk is a kind of credit risk among the associated credit entities formed by credit-related entities. Focusing on this hot topic of associated credit risk and the relevant contagion and considering the latent entities and their incubatory period, this paper builds an infectious dynamic model to describe the associated credit risk contagion of associated credit entities based on the mean-field theory of complex networks. Firstly, this paper analyzes the stable state of the associated credit risk contagion in the associated entity network, considering the latent entities and their incubatory period. Secondly, from the perspective of complex network and considering the incubatory period, a SHIS model is built to reveal how the incubatory period influences associated credit risk contagion. Finally, the sensitivity of some parameters is analyzed in the Barabási-Albert (BA) scale-free network. The results show the following: (i) the contagion threshold of associated credit risk is related to the incubatory period of latent entities, the recovery rate and infectivity of infected entities, and the newborn rate of credit entities; (ii) the infectious rate of infected entities, the mortality rate of credit entities, and the important factors stated in (i) are all significantly correlated with the density of infected entities.
\end{abstract}

\section{Introduction}

At present, the concept of credit risk has not formed a unified definition [1]. The conventional view is that credit risk refers to the risk that the counterparty or borrower is unable or unwilling to perform the contract; that is, the possibility that the default caused by the debtor's failure to repay the debts in the contract on time will bring losses to the creditors [1]. Nowadays, the world economic development has entered a new stage, and the credit relationship among enterprises, banks, insurance companies, and other credit entities is becoming increasingly closer and more extensive, thus forming associated credit entities. If the default of some credit entities increases the defaults or the default probability of the other credit entities that are associated with them, this credit risk is then called the associated credit risk among the associated credit entities [2]. It can be seen that the associated credit risk is a special kind of credit risk.

The U.S. subprime mortgage crisis spread rapidly to many countries around the world and eventually led to the bankruptcy or reorganization of several well-known investment banks, commercial banks, and insurance companies, resulting in turmoil in the entire financial market in 2008. The phenomenon appears that some subprime 
borrowers have failed to repay their loans on time in the early spring of 2006, and this subprime mortgage crisis swept through major financial markets in the world, such as the United States and the European Union in August 2007. So the incubatory period of associated credit risk is noticeable. Affected by above subprime crisis, Greece's sovereign credit was downgraded, causing the European debt crisis to erupt and spread rapidly throughout the euro area at the end of 2009. It lasts for a long time from subprime crisis to European debt crisis, and these two big finance crises both cause significant fluctuations and losses of the global economy, which are both called systemic risk according to the literature [3]. The core of systemic risk concern is contagious [4]. Credit risk is a kind of nonsystemic risk, the core of credit risk concern is the credit entities' default, and the credit risk is also contagious [4]. If an entity does not pay enough attention to the incubatory period of associated credit risk and do not guard against credit risk in the incubatory period, it is likely that systemic financial risk will be caused by credit risk contagion, and just like above subprime crisis and European debt crisis mentioned, the regional economy and even the global economy will be severely damaged. Therefore, when identifying the associated credit risk and exploring the evolutionary law of associated credit risk contagion, the incubatory period of the associated entity should be considered.

For the evolutionary mechanism of the associated credit risk contagion, the above studies may be more reasonable if considering the latent entities and incubatory period. In fact, in the early stage of the contagion and evolution of associated credit risk, some credit entities have high credit levels including strong operational capability, highly profitable and liquidity, and reasonable governance structures. These credit entities have a specific resistance to the associated credit risk, which may have been infected, but the symptoms of the credit risk have not been shown, or they are unwilling to disclose or even intentionally hide their own credit risk for themselves. This period of hiding credit risk is called the incubatory period. However, with the increase in the ability of the associated credit risk to infect the associated entities and the change in the internal and external environment of the credit entities, the risk incubatory period of the latent entities may come to an end, and they may become infected entities with high credit risk. After effective control and management, such as rescue or immunization, the infected entities decrease their own credit risk, which may become susceptible entities again. Credit risk evolves in the associated entity network.

To summarize, in this paper, based on the classical SIS epidemiological model, a SHIS derived model is constructed to study associated credit risk contagion in an associated entity network to reveal the influence of incubatory period and relevant parameters on credit risk contagion. The highlights of this paper are to make use of the SHIS infectious dynamics model and introduce latent entities and the incubatory period to characterize the evolutionary contagion mechanism of associated credit risk in the associated entity network.
The remainder of this paper is organized as follows: Section 2 is an analysis of epidemiological model applied to associated credit risk contagion; Section 3 is a literature overview; Section 4 is a description of the basic epidemiological model; Section 5 includes assumptions, derived epidemiological model construction, and theoretical analysis; and Section 6 presents our simulation analysis of the derived epidemiological model. By using simulation software, we analyze the influence of different factors on associated credit risk contagion, including incubatory period. Section 7 is the conclusion of this paper and the prospects for future work.

\section{Feasibility Analysis of Epidemiological Model Applied to Associated Credit Risk Contagion}

May et al. find that financial risk contagion is similar to the epidemic spread, so the epidemiological model is used to describe the contagion of financial risk [5]. Luo et al. and $\mathrm{Xu}$ et al. also pointed out that risk contagion in the guarantee network is similar to the virus spread and briefly described similarity characteristic between them $[6,7]$. Based on the previous research results, combined with the infectious evolution characteristics of the associated credit risk, we find that the infectious evolution of the associated credit risk in the associated entity network has many similarities with the spreading of the epidemic virus in the social network. For example, there is being infectious source, infectivity, immunity, and reversibility of circulation.

The evolution process of epidemic virus is similar to that of associated credit risk, which shows two kinds of characteristics: one is the similarity of infectious structure, and the other is the similarity of infectious mode. The infectious structure includes diffusion channels and infection targets, and the infectious mode includes infectious radiation and infectious process. The details are listed as follows.

The infectious structure is similar. The epidemic virus spreads in the social group network composed of individuals, the spreading channel is the path of the social group network which is the mutual relationship between individuals, and the spreading goal of the epidemic virus is the individual node of the social group network. However, the contagion of associated credit risk is spreading in the associated entity network, the spreading channel is the direct or indirect relationship between the associated entities, and the spreading goal of the associated credit risk is the node of associated entity network. Furthermore, due to the difference in individual autoimmunity in the social group network, the ability of resisting virus invasion is different. However, due to the difference in governance level (including operation, financing, profitability, and solvency) of associated entities in the associated entity network, the ability of resisting associated credit risk is also different. Therefore, in terms of infection structure, associated credit risk and epidemic virus are similar.

The infectious mode is similar. The spread of epidemic virus in the social group network takes the shape of radiation, while the associated entities with credit risk have radiation negative effects on other associated entities in the associated entity network. In addition, the virus generally 
spreads through contacts in the contagion process of epidemic, while in the contagion process of associated credit risk, a node entity with credit risk is also preferred to have negative effects on the associated entity that has direct relationship with the infected node entity. Therefore, in terms of infectious mode, associated credit risk and epidemic virus are similar.

\section{Literature Overview}

This paper is closely related to three strands of the existing literature. First, our paper is linked to work on financial network and contagion. Second, our analysis is closely related to work on epidemiological model and credit risk contagion. The third strand of relevant literature investigates the associated credit risk contagion and relevant delayed effects.

First, this paper is linked to existing work on financial network and contagion. Elliott et al. studied the financial contagions and cascades of failures affected by integration and diversification in a network of financial interdependencies [8]. Based on susceptible-infected-recovered (SIR) model, Fisher established the financial network contagion model and found that shocks in this financial market exhibit complex evolutionary dynamics that either tend to increasingly fragile states or the elimination of a high number of competitors, detrimental to a more decentralized market order [9]. Unlike Fisher, Eboli represented a financial system as a flow network and model the process of direct balancesheet contagion as a flow of losses crossing such a network and focused most of the analysis on the effects that the connectivity and centralization of a financial network have on its exposure to default contagion and then found that both the complete and the star networks show a robust yet fragile response to shocks [10]. Gencay et al. discussed that network uncertainty gives rise to an endogenous core-periphery structure which is optimal in mitigating financial contagion yet concentrates systemic risk at the core of big banks [11]. Dastkhan and Gharneh introduced a simulation model to analyze the contagion in financial markets based on the cross shareholding network of firms and the probability and the extent of contagion [12]. Bucci et al. developed an epidemiological approach to analyze the mutual influence between financial contagion and economic activity and showed that financial contagion in some specific regions may propagate quickly also in regions far away from those in which the contagion initially occurs, highlighting the role of regional policy coordination to avoid interregional contagion [13]. Hu and Li put epidemiological model into scalefree financial network and discuss the influence of infection rate on financial risk contagion [14].

In order to prevent financial and economic crisis, Dastkhan applied the forward-looking conditional value at risk (COVaR) as a market-based systemic risk measure to analyze the systemic risk in an emerging market and predict financial crisis [15]. However, Sarlin evaluated the performance of biologically inspired early warning systems for systemic financial crises based on a logit model, a standard back-propagation neural network, and a neurogenetic model
[16]. Giudici et al. applied correlation network models that tap into the multivariate network structure, as a viable means to assess common exposures and complement direct linkages, and found that common exposures indeed are channels of contagion and should be accounted for when measuring systemic risk [17]. Apergis et al. found contagion occurred during the recent global financial crisis across European and US financial markets through the channels of higher order moments [18].

Second, this paper is closely related to work on epidemiological model and credit risk contagion. Chakrabarty and Zhang examined the negative consequences of the contagion effect using variables, such as the number of transactions, the size of transactions, and the volume of transactions [19]. Jorion et al. found that affiliated transactions lead to credit risk contagion among enterprises, and counterparty default is the potential cause of credit risk contagion [20]. Through numerical simulation, the contagion mechanism caused by the business relationships among enterprises in bank loan portfolios was studied [21]. Based on the epidemiological model, a network model is built with consideration of investor behavior and information disclosure strategy, and the mechanism of counterparty credit risk contagion is analyzed [22]. A counterparty credit risk contagion network evolution model SIRS is developed to study counterparty credit risk contagion in the CRT market and its influencing factors [23]. Chen et al. studied the causes, mechanisms, and control strategies of credit risk contagion among CDS counterparties and found that information asymmetry and innovation diffusion could cause credit risk contagion among CDS counterparties [24]. Later, Chen et al. introduced an evolving network model of credit risk contagion in the credit risk transfer (CRT) market and described the influence and active mechanism of the spillover effects of infected investors, behaviors of investors and regulators, emotional disturbance of investors, market noise, and CRT network structure on credit risk contagion [25]. However, $\mathrm{Li}$ et al. studied the credit risk with an affiliated guarantee relationship and found that the contagion intensity of credit risk was positively correlated with the proportion of equity [26]. Yang and $\mathrm{Hu}$ studied the risk contagion model of China's interbank market, characterized the interbank network structure by using a core-edge network, and found that the ability of Chinese banks to withstand risks has improved in recent years [27]. Wu et al. found that changes in bank credit ratings can cause risk contagion within the bank network that is composed of interbank lending relationships [28]. Based on the SIR model, Wang et al. built a network model with the consideration of investor behavior and information disclosure strategy and analyzed the mechanism of counterparty credit risk contagion [29]. Jiang et al. proposed two credit risk contagion models separately considering asymmetric information association and sentiment contagion and further analyzed the influence of network structure, information association, individual risk attitude, financial market supervision intensity, and individual risk resisting ability on individual risk contagion $[23,30]$. It can be seen that the above-mentioned counterparty risk, correlation, 
information asymmetry, authenticity, sentiment, and network structure trigger the credit risk contagion. Besides, Giusy et al. investigated the relationship between exposure to climate change and firm credit risk and indicated that the exposure to climate risks affects the creditworthiness of loans and bonds issued by corporates [31].

A lot of literature has emerged on sovereign credit default contagion. De Bruyckere et al. investigated contagion between bank and sovereign default risk in Europe over the period 2007-2012, and empirical certified that three contagion channels are at work: a guarantee channel, an asset holdings channel, and a collateral channel [32]. Breckenfelder and Schwaab studied spillovers from bank to sovereign risk in the euro area and surprisingly found that bank risk in stressed countries was not absorbed by their sovereigns but spilled over to nonstressed euro area sovereigns [33]. Greenwoodnimmo et al. developed an empirical network model to study credit risk spillovers among a group of eighteen sovereigns and their financial sectors from 2006 to 2015 [34]. Srivastava et al. applied an error correction model to document strong evidence of Granger causality in mean from the S\&P option market to the sovereign CDS market in $98 \%$ of the 56 sovereigns [35]. Bostanci and Yilmaz studied the network structure of the volatility of the sovereign credit default swaps spreads and found that both trade and capital flows are important determinants of pairwise connectedness across countries [36].

Finally, this work is closely related to literature on associated credit risk contagion and relevant delay effect. Xu et al. combined an epidemic model and complex network theory and explored the impacts of risk information and individual responses on associated credit risk [37]. Based on the improved epidemiological model, Qian et al. studied the stable state characteristics of the associated credit risk contagion in immunization scenarios [38]. Based on the theory of infectious diseases, $\mathrm{Li}$ et al. established a stochastic dynamic model of credit risk contagion in enterprise groups and analyzed the stable condition of the model and the arrival time of default peaks [39]. Qian et al. constructed a dual-layer network and found that entrepreneurs' spreading willingness and the authenticity of information will affect the associated credit risk contagion [40]. The above literature research lays the theoretical foundation for this paper to use an epidemiological model and complex network theory to study the associated credit risk contagion.

About the delayed effects in complex networks, many studies related to viruses or infectious diseases are often presented. These literatures consider delay or incubatory period and study the propagation dynamics properties of virus including threshold, global stability, and immune strategy [41-45], which inspires us to study the credit risk contagion with incubatory period. Viviana and Lucia proposed a particular time-delay susceptible-infected-recovered model to investigate and describe the credit risk contagion in the market and studied the steady states according to different values of time delay and different bank support policies [46]. Li et al. studied the delayed effect of the contagion of associated credit risk in scale-free and smallworld networks and further explored the impact of immune failure and immune invalidity on the contagion of the associated credit risk under incomplete immunization [2, 47, 48]. Based on above literatures, we consider the incubation period to study the associated credit risk contagion in the associated entity network.

\section{Basic Epidemiological Model}

Many social, biological, and communication systems can be properly described by complex networks whose nodes represent individuals or organizations and links mimic the interactions among them $[49,50]$. The following main approaches were borrowed from biological epidemiology. The standard model used in the study of computer virus infections is the susceptible-infected-susceptible (SIS) epidemiological model [51]. In this SIS model, each node of the network represents an individual and each link is a connection along which the infection can spread, and individuals exist only two discrete states: "healthy" or "infected." At each time step, each susceptible (healthy) node is infected at rate $v$ if it is connected to one or more infected nodes. Infected nodes are cured and become again susceptible at rate $\delta$, defining an effective spreading rate $\lambda=\nu / \delta$. Without loss of generality, we can set $\delta=1$. This SIS model does not take into account immunization, and individuals run stochastically through the cycle susceptible $\longrightarrow$ infected $\longrightarrow$ susceptible. Thus, based on the SIS epidemiological model and literature [51], the dynamical mean field equation (1) is established as

$$
\left\{\begin{array}{l}
\frac{d S_{k}(t)}{d t}=I_{k}(t)-\lambda k\left[1-I_{k}(t)\right] \Theta\left(I_{k}(t)\right), \\
\frac{d I_{k}(t)}{d t}=-I_{k}(t)+\lambda k\left[1-I_{k}(t)\right] \Theta\left(I_{k}(t)\right),
\end{array}\right.
$$

where density $I_{k}(t)$ of infected nodes with given connectivity $k$, i.e., the probability that a node with $k$ links, is infected [51]. The first formula of equation (1) considers infected nodes becoming healthy with probability $\delta=1$. The second formula of equation (1) represents the density of newly generated infected nodes that is to the infection rate $\lambda$ and the probability that a node with $k$ links is healthy $\left[1-I_{k}(t)\right]$ and obtains the infection via a connected node [38]. $\Theta\left(I_{k}(t)\right)$ with any given link points to an infected node, which can be written as

$$
\Theta\left(I_{k}(t)\right)=\frac{1}{\langle k\rangle} \sum_{k} k P\langle k\rangle I_{k}(t) .
$$

Through solving equations (1) and (2) in the stationary state $\left[d I_{k}(t) / d t=0\right]$, the below self-consistency (3) is obtained [38] as

$$
\Theta\left(I_{k}(t)\right)=\frac{1}{\langle k\rangle} \sum_{k} k P\langle k\rangle \frac{\lambda k \Theta\left(I_{k}(t)\right)}{1+\lambda k \Theta\left(I_{k}(t)\right)},
$$

where $\Theta\left(I_{k}(t)\right)$ is a function of $\lambda$. The self-consistency equation (3) allows the condition $\lambda\left\langle k^{2}\right\rangle /\langle k\rangle \geq 1$ only if 
$\Theta\left(I_{k}(t)\right) \neq 0$ and $I_{k}(t) \neq 0$, so the epidemic threshold is defined as

$$
\lambda_{c}=\frac{\langle k\rangle}{\left\langle k^{2}\right\rangle},
$$

where $\left\langle k^{2}\right\rangle$ is the second moment of degree distribution. If $\lambda$ is above the threshold, i.e., $\lambda \geq \lambda_{c}$, the infection spreads and becomes possible. Otherwise, $\lambda \leq \lambda_{c}$, and the infection dies out exponentially [51].

\section{Derived Epidemiological Model}

5.1. Assumptions. For the convenience of the research, this paper proposes the following assumptions based on the characteristics of the contagion evolution of associated credit risk.

Assumption 1: In the network composed of $N$ associated entities, the associated entities exist only in three discrete states, "susceptible," "latent," or "infected," which are divided into three categories: susceptible entities, latent entities, and infected entities.

(1) Susceptible entity $S$ : in the associated entity network, the susceptible entity refers to the entity with a lower credit risk that is vulnerable to be infected by the high credit risk entity associated with it, thus increasing the possibility of becoming a high credit risk entity. This kind of credit entity's own credit risk is at a low level, but it is easy to be infected by the high credit risk entity, and it is easier to be infected by the high credit risk entity closely related to it. For example, those enterprises and financial institutions with excessive financial leverage, high asset-liability ratio, and temporary shortage of cash flow are vulnerable to be infected by the credit risk because their ability to resist credit risk is weakened. There are a large number of them in the network of associated entities. Once infected, some susceptible entities need self-protection and operation and are unwilling to disclose or even intentionally hide their real credit risk. This period of hiding their own real credit risk is called incubatory period. These susceptible entities in incubatory period are transformed into latent entities.

(2) Latent entity $\mathrm{H}$ : in the associated entity network, the latent entity refers to the entity which has been infectious, and its credit risk is at a little high level but cannot infect other associated entities. That is, this kind of credit entity cannot enlarge the credit risk of other credit entities through the relationship between them. For example, those enterprises and financial institutions with profit margin remained at a high level, high accounts receivable, and high debt ratio, which have weak linkages with other susceptible entities. Under the influence of own internal factors and external macroenvironment, after some time $\tau$ ( $\tau \geq 0$, incubatory period), such entities with high credit risk are transformed into infected entities.

(3) Infected entity I: in the associated entity network, the infected entity refers to the entity which has been infectious, and its credit risk is at an extremely high level and can infect the associated entities. That is, this kind of credit entity can magnify the credit risk of other credit entities through the relationship between them. The change in the proportion in the network indicates the contagion effect of associated credit risk. For example, those enterprises and financial institutions have suffered from severe operational problems such as long-term losses, insolvency, and serious shortage of cash flow.

Assumption 2: A susceptible entity can be changed into a latent entity at an invariant probability $\lambda$ through the correlation with an infected entity. In this network, before a susceptible entity can be changed into an infected entity, it must undergo an incubatory period, and the latent entity can only be changed into an infected entity. Here, $\lambda$ is the contagion probability $(0<\lambda \leq 1)$ of associated credit risk, which expresses the speed of contagion and diffusion of the associated credit risk in the network of associated entities. Infected entities are not immune and become susceptible entities after being cured or recovered. At this moment, if the susceptible entities contact an infected entity because of the relationship, they will be infected with a certain probability $\lambda$.

Assumption 3: An infected entity will be cured at a rate of $\delta$ and become susceptible entity, where $\delta$ is the recovery rate $(0<\delta \leq 1)$.

An associated entity network that is composed of associated entities is always dynamically changing. There are usually credit entities entering the network, and there will also be credit entities that naturally disappear or leave the network. In general, there is the following assumption.

Assumption 4. A credit entity that enters the network anew is called a newborn, and the new rate is $M$. Assume this newborn entity is susceptible. A credit entity that naturally disappears or withdraws from the network is dead, and the death rate is $m$. Suppose this dead entity is infected entity. The associated entity network is in the process of continuous development and expansion and so $0<m \leq M<1$.

5.2. Derived Model Construction. In a complex network of associated entities that is composed of $N$ associated credit entities, the credit entities are nodes in the network, and the relationships among the nodes are the edges of the network. The degree $k_{i}$ of node entity $i$ is defined as the number of edges that are connected to the entity. The average of the degree $k_{i}$ of all nodes in the network is called the network average, and it is denoted as $\langle k\rangle$. Denote $P\left(k_{i}\right)$ as the probability of randomly selecting a node $i$ with degree $k_{i}$ and so $\langle k\rangle=\sum_{i=1}^{n} k_{i} P\left(k_{i}\right)$. The densities of susceptible entities, 
latent entities, and infected entities at time $t$ and degree $k$ are expressed as $S_{k}(t), H_{k}(t)$, and $I_{k}(t)$, respectively, and here, the density is the proportion. Therefore,

$$
S_{k}(t)+H_{k}(t)+I_{k}(t)=1 \text {. }
$$

$P(t)$ denotes the probability that a credit entity is still in the incubatory state within period $t$ after it enters the incubatory state (without considering death), and therefore, $\lim _{t \rightarrow \infty} P(t)=0, \lim _{t \rightarrow 0} P(t)=1$, and $\int_{0}^{\infty} P(v) d v$ is a finite positive number.

Considering the actual situation of enterprise type, nature, strength, and operation management, there are two typical situations. The first is that there are susceptible entities (S), latent entities $(\mathrm{H})$, and infected entities (I) in the associated entity network. The second is that there are susceptible entities $(S)$, latent entities $(H)$, infected entities (I), and recovery entities (R) in the associated entity network. The first typical situation is studied in this paper, in which situation, S, H, and I are defined in Section 5.1.

It is assumed that there are only susceptible entities $(S)$ in the associated entity network at the beginning. With the change in external objective macroenvironment and internal subjective factors, some susceptible entities with highly profitable and liquid and reasonable governance structures may be infected, but these credit entities have some specific resistance to the associated credit risk, and the symptoms of the credit risk have not been shown, which are called hidden entities $(\mathrm{H})$. However, with the increase in infectious capacity of associated credit risk and the change in the internal and external environments of the credit entities, hidden entities may become infected entities (I). After effective control and management, such as rescue or immunization, the infected entities may get out of trouble and decrease their own credit risk, which may become susceptible entities (S) again. Credit risk evolves in the associated entity network. Therefore, the epidemiological model describing this kind of station is the susceptible $\longrightarrow$ incubatory $\longrightarrow$ infected $\longrightarrow$ susceptible (SHIS) model.

According to the above basic epidemiological model, hypothesis, and using dynamic mean-field theory, the first equation of the SHIS model can be described as

$$
\frac{d S_{k}(t)}{d t}=M-\lambda k S_{k}(t) \Theta(t)-m S_{k}(t)+\delta I_{k}(t),
$$

where $M$ is described in Assumption $4, \lambda k S_{k}(t) \Theta(t)$ denotes the decrease of susceptible entities due to infection, $m S_{k}(t)$ denotes the decrease of susceptible entities due to death, and $\delta I_{k}(t)$ denotes the increase of susceptible entities that come from infected entities due to treatment.

The second equation of SHIS model can be described as

$$
H_{k}(t)=\int_{0}^{t} \lambda k S_{k}(\eta) \Theta(\eta) e^{-M(t-\eta)} P(t-\eta) d \eta,
$$

where $H_{k}(t)$ denotes the number of susceptible entities entering into latent entities due to infection and $e^{-z t}$ denotes the probability that a credit entity is still in the incubatory state within period $t$ after it enters the incubatory state (without considering death). $\Theta(t)$ denotes that any given link points to an infected node at time $t$, and the degreeindependent network is considered in this paper. Therefore, $\Theta(t)$ can be written as

$$
\Theta(t)=\frac{1}{\langle k\rangle} \sum_{i=1}^{n} \varphi(i) P(i) I_{i}(t)
$$

where $\varphi(k)$ is the infectivity function of the nodes with degree $k$.

Equation (7) is the Riemann-Stieltjes integral, and so

$$
\begin{aligned}
\frac{d H_{k}(t)}{d t}= & \lambda k S_{k}(t) \Theta(t) \\
& +\int_{0}^{t} \lambda k S_{k}(\eta) \Theta(\eta) e^{-M(t-\eta)} M_{t} P(t-\eta) d \eta-M H_{k}(t) .
\end{aligned}
$$

The third equation (9) of SHIS model can be described as $\frac{d I_{k}(t)}{d t}=\int_{0}^{t} \lambda k S_{k}(\eta) \Theta(\eta) e^{-M(t-\eta)} M_{t} P(t-\eta) d \eta-(\delta+M) I_{k}(t)$.

Simplifying equation (10), we get

$$
\frac{d I_{k}(t)}{d t}=\lambda k e^{-M \tau} S_{k}(t-\tau) \Theta(t-\tau)-(\delta+M) I_{k}(t)
$$

where $\lambda k e^{-M \tau} S_{k}(t-\tau) \Theta(t-\tau)$ denotes the number of latent entities entering into infected entities due to infection, $\delta I_{k}(t)$ denotes the decrease of infected entities due to treatment, and $M I_{k}(t)$ denotes the decrease due to the newborn.

The SHIS model is complex and challenging to study. Therefore, only the probability function $P(t)$ is taken as the following state function:

$$
P(t)= \begin{cases}1, & t \in[0, \tau] \\ 0, & t>\tau\end{cases}
$$

where $t>\tau$, obtained from equation (9) as

$$
\frac{d H_{k}(t)}{d t}=\lambda k S_{k}(t) \Theta(t)-\lambda k e^{-M \tau} S_{k}(t-\tau) \Theta(t-\tau)-M H_{k}(t),
$$

where $0 \leq t \leq \tau$, obtained from equation (9) as

$$
\frac{d H_{k}(t)}{d t}=\lambda k S_{k}(t) \Theta(t)-M H_{k}(t) .
$$

5.3. Theoretical Analysis. Equations (6), (7), and (10) depict the transformation relationship among the susceptible entities, latent entities, and infected entities. After a period of contagious evolution, if the contagion of the associated credit risk in the associated entity network is in a long-term disordered and uncertain state, the associated entity network is considered to be in an unstable state. Conversely, if the contagion of the associated credit risk tends to be stable, that is, the densities of the three types of entities in the associated entity network tend to be stable, the associated entity 
network is considered to be in a stable state. This paper focuses on this stable state, that is, the stable risk state. Furthermore, in this stable risk state, the density of infectious agents in the associated entity network tends to be constant.

Therefore, in order to conduct the research conveniently, when $t>\tau$, this paper only discusses the following equation:

$$
\frac{d I_{k}(t)}{d t}=\lambda k e^{-M \tau} S_{k}(t-\tau) \Theta(t-\tau)-(\delta+M) I_{k}(t) .
$$

The analytic solution of equation (15) is not easy to find, and the solution under a stable risk state is considered instead. The densities of the susceptible entities and infected entities with a degree $k$ in the stable state are $S_{k}^{*}$ and $I_{k}^{*}$, respectively; $S^{*}$ and $I^{*}$ are the densities of susceptible entities and infected entities in the whole associated entity network in stable state, respectively. The probability of a random edge being connected to an infected entity in a stable state is $\Theta^{*}$.

Definition 1. When the contagion probability $\lambda$ of the associated credit risk is less than a specific probability value $\lambda_{c}$, the associated credit risk is no longer contagious in the associated entity network, where $\lambda_{c}$ is called the contagion threshold of the associated credit risk in the associated entity network.

Proposition 1. The contagion threshold of the associated credit risk considering the incubatory period is $\lambda_{c}=(\delta+M)\langle k\rangle e^{M \tau} /\langle\varphi(k)\rangle$. When $\lambda<\lambda_{c}$, the associated credit risk is no longer contagious in the associated entity network, and equations (6), (7), and (10) converged to the equilibrium point $(M / m, 0)$; when $\lambda>\lambda_{c}$, equations (6), (7), and (10) converged to the equilibrium point $\left(S^{*}, I^{*}\right)$.

Proof. In the stable state, $d S_{k}(t) / d t=0$ and $d I_{k}(t) / d t=0$, and the solution of equations (6) and (11) in the stable state is obtained as

$$
\left\{\begin{array}{l}
S_{k}^{*}=\frac{M+\delta I_{k}^{*}}{\lambda k \Theta^{*}+m}, \\
I_{k}^{*}=\frac{\lambda k M \Theta^{*} e^{-M \tau}}{(\delta+M)\left(\lambda k \Theta^{*}+m\right)-\lambda k \delta e^{-M \tau} \Theta^{*}},
\end{array}\right.
$$

where $\Theta^{*}=(1 /\langle k\rangle) \sum_{i=1}^{n} \varphi(i) P(i) I_{i}^{*}$.

The following formula of equation (16) is put into equation (8), where $\Theta^{*}$ satisfies the self-consistency condition, obtained as

$$
\Theta^{*}=\frac{1}{\langle k\rangle} \sum_{i=1}^{n} \varphi(i) P(i) \frac{\lambda i M \Theta^{*} e^{-M \tau}}{(\delta+M)\left(\lambda i \Theta^{*}+m\right)-\lambda i \delta e^{-M \tau} \Theta^{*}}=f(\Theta) .
$$

$\Theta^{*}=0$ is a trivial solution of equation (11). Besides, $f(\Theta)$ is continuously differentiable, obtained as

$$
f^{\prime}(\Theta)=\sum_{i=1}^{n} \varphi(i) P(i) \frac{M^{2} e^{M \tau}}{\langle k\rangle \lambda i(\delta+M) \delta^{2} \Theta^{2}(\lambda i \Theta+m)^{2}}>0 .
$$

$f(\Theta)$ increases with $\Theta$. When $f^{\prime}(\Theta) \mid \Theta_{=0}>1$, namely, $\lambda e^{-M \tau}\langle\varphi(k)\rangle /(\delta+M)\langle k\rangle>1$, and there is a nontrivial solution to equation (17) within $0<\Theta^{*}<1$, and the contagion threshold is obtained as $\lambda_{c}=(\delta+M)\langle k\rangle e^{M \tau} /\langle\varphi(k)\rangle$. When this nontrivial solution $\Theta^{*}$ is put into (16), $S_{k}^{*}$ and $I_{k}^{*}$ are both obtained. Because $S^{*}=\sum_{k} P(k) S_{k}^{*}$ and $I^{*}=\sum_{k} P(k) I_{k}^{*}$, the equilibrium point $\left(S^{*}, I^{*}\right)$ exists.

From the expression of $\lambda_{c}$, it is found that the contagion threshold of associated credit risk is not only related to the topological structure of the network but also related to the recovery rate of the infected entity, the newborn rate of the credit entity, the incubatory period of the latent entity, and the contagion ability function of the infected entity. As the recovery rate increases, the contagion threshold of the associated credit risk increases. As the freshness of the credit entity increases, the contagion threshold of the associated credit risk increases. As the incubatory period of the possible entity increases, the contagion threshold of associated credit risk increases. Finally, as the contagion ability of the infected entity increases, the contagion threshold of associated credit risk decreases. In next section, more detailed discussion will be studied by simulation analysis under some assumptions.

\section{Simulation Analysis}

In this section, the simulations are analyzed in BA scale-free network. The associated entity network is a complex system of multientities, multilinks, multitasks, self-organization, and self-learning. In recent years, with the evolution of the macroenvironment, such as market and microindividual factors, the associated entity network has presented complex network characteristics. In the associated entity network, there are a few entities that are connected with more related entities; there are also situations in which most entities are only connected with other minority entities, that is, preferential connectivity; and most of the real-world network scale is expanding, that is, growth. Therefore, the network presents scale-free characteristics. The existing literature shows that scale-free networks can accurately depict the characteristics of interbank overnight lending networks, intercountry banking networks, and different stock market networks [5-7] that can be used to study the contagious characteristics of associated credit risks $[26,27,52]$. In view of this, this paper chooses the BA scale-free network to analyze the contagious evolutionary law of the credit risk of associated entities. In the BA scale-free network, if $k$ is continuous, the network's average degree is $\langle k\rangle=\int_{w}^{+\infty} k P(k) d k=2 w$, and the node degree distribution is $P(k) \stackrel{w}{=} 2 w^{2} k^{-3}$, where $w$ is the smallest degree of credit entity in the network.

Concerning the contagion ability function $\varphi(k)$, in order to get close to the real risk contagion network and better study the infectious characteristics of associated credit risk, the assumption of piecewise linear infectivity is adopted to describe $\varphi(k)$ in this paper [18], namely, $\varphi(k)=\min (\alpha k, A)$. When the degree $k$ of an entity node is small, its contagion capacity is proportional to $k$, which is $\varphi(k)=\alpha k$. When $k$ is overwhelmingly large and over one constant $A / \alpha$, its 
contagion capacity is $A$, namely, $\varphi(k)=A$. Here, $A>0$ is a constant parameter where $0<\alpha \leq 1$.

In $B A$ scale-free networks, when $\varphi(k)=\alpha k$, the contagion threshold of associated credit risk is as

$$
\lambda_{c}=\frac{(\delta+M)\langle k\rangle e^{M \tau}}{\langle\varphi(k)\rangle}=\frac{(\delta+M) e^{M \tau}}{\alpha},
$$

where $\varphi(k)=A$. The contagion threshold of associated credit risk is as

$$
\lambda_{c}=\frac{(\delta+M)\langle k\rangle e^{M \tau}}{\langle\varphi(k)\rangle}=\frac{2(\delta+M) w e^{M \tau}}{A P(A)},
$$

where $P(A)>0$ is a constant and $A P(A)>0$ is also a constant. To simplify the research, the network in the real world is limited, so $\varphi(k)=\alpha k$.

From the expression $\lambda_{c}$ of equations (19) and (20), it is found that the contagion threshold of associated credit risk is related to contagion ability function, the recovery rate of the infected entity, the new rate, and the incubatory period of the latent entity.

The node distribution $P(k)$ and average degree $\langle k\rangle$ of the $B A$ scale-free networks are both put into (17), and the following is obtained:

$$
2 w\left[(\delta+M)\left(\lambda i \Theta^{*}+m\right)-\lambda i \delta e^{-M \tau} \Theta^{*}\right]=\lambda i M e^{-M \tau} \int_{1}^{n} \frac{2 \alpha w^{2}}{i^{2}} d i .
$$

Inserting equation (20) into equation (21), the analytical solution is obtained:

$$
\Theta^{*}=\frac{\lambda \alpha M w i e^{-M \tau}-m(\delta+M)}{\lambda i\left(\delta+M-\delta e^{-M \tau}\right)} .
$$

Inserting equation (22) into equation (16), we can get

$$
I_{k}^{*}=\frac{(\delta+M) \lambda m}{M w \alpha\left(\delta+M-\delta e^{-M \tau}\right) k}+\frac{M}{e^{M \tau}(\delta+M)-\delta} .
$$

Therefore,

$$
I^{*}=\sum_{k} P(k) I_{k}^{*}=\frac{2 w(\delta+M) \lambda m}{3 M \alpha\left(\delta+M-\delta e^{-M \tau}\right)}+\frac{M w^{2}}{e^{M \tau}(\delta+M)-\delta} .
$$

Obviously, in BA scale-free networks, in addition to the network structure, the density of the infected entities in the whole network is also related to the new rate, mortality rate, incubatory period of the latent entities, recovery rate of the immune entities, infection rate, and infection coefficient of the infected entities. Moreover, when $\lambda<\lambda_{c}$, the associated credit risk is not contagious. When $\lambda>\lambda_{c}$, the associated credit risk is contagious. Therefore, the contagion effect of associated credit risk exists in associated entity networks.

6.1. Comparative Analysis without considering the Incubatory Period. Without considering the incubatory period, that is $\tau=0$, the contagion threshold of associated credit risk is $\lambda_{c}^{0}=(\delta+M) / \alpha$, and the overall density of infected entities in the stable state is $I^{*^{0}}=\left(2 w(\delta+M) \lambda m / 3 M^{2} \alpha\right)+w^{2}$.
Considering the incubatory period, that is, $\tau>0$, the contagion threshold of associated credit risk is $\lambda_{c}=(\delta+M) e^{M \tau} / \alpha$, and the overall density of infected entities in the stable state is $I^{*}=\sum P(k) I_{k}^{*}=(2 w(\delta+$ $\left.M) \lambda m / 3 M \alpha\left(\delta+M-\delta e^{-M \tau}\right)\right)+\left(M w^{2 k} / e^{M \tau}(\delta+M)-\delta\right)$.

Comparatively, $\lambda_{c}>\lambda_{c}^{0}$, which means that the contagion threshold of associated credit risk will be underestimated without considering the incubatory period, and $I^{*}<I^{*^{0}}$, which means that the density of infected entities in the stable state may be overestimated without considering the incubatory period. Refer to the parameters value of literature $[37,38,40,47,52]$, parameter values are given in the below numerical simulation.

Figure 1 shows that the interaction of the infected entities density $I^{*}$, the incubatory period $\tau$, and the infection probability $\lambda$ in the stable state when $\alpha=0.1, w=2$, $M=0.05, m=0.01$, and $\delta=0.5$. The density of infected entities without considering the incubatory period always outweighs the density of infected entities when considering the incubatory period, that is, $I^{* 0}>I^{*}$.

6.2. Numerical Simulation and Analysis of the Density of Infected Entities. The interaction between the density of infected entities $I$, the incubatory period of latent entities $\tau$, the infection probability $\lambda$, the infectivity coefficient $\alpha$, the recovery rate of infected entities $\delta$, the new rate of credit entities $M$, and the mortality rate of credit entities $m$ in the BA scale-free network can be calculated.

It can be seen from Figure 2 that the changing relationship between the density of infected entities $I$ and the incubatory period $\tau$ of latent entities considering different infection probabilities of infected entities $\lambda_{1}=0.1$, $\lambda_{2}=0.3$, and $\lambda_{3}=0.5 \quad$ when $\quad \alpha=0.1, w=2, M=0.05$, $m=0.01$, and $\delta=0.5$. As can be seen from Figure 2, (i) when the infection probability of infected entities remains unchanged, as the incubatory period of latent entities increases, the infectivity of the associated credit risk in the network decreases, and increasingly fewer associated entities are infected, which is consistent with the reality. (ii) When the incubatory period is unchanged, as the infection probability increases, the contagion effect of the associated credit risk in the network increases, and so the density of infected entities in the network will increase. That is, the number of infected entities in the network will increase as the contagion of the associated credit risk increases, which is consistent with reality. It can be seen that the incubatory period of latent entities and the infection probability of infected entities are important factors affecting the density of infected entities.

Figure 3 shows the changing relationship between the density of infected entities $I$ and the incubatory period $\tau$ of latent entities considering different recovery rates of infected entities $\delta_{1}=0.4, \delta_{2}=0.6$, and $\delta_{3}=0.8$ when $\alpha=0.1, w=2$, $M=0.05, m=0.01$, and $\lambda=0.1$. The following can be seen from Figure 3: (i) when the recovery rate of infected entities remains unchanged, as the incubatory period of latent entities increases, the infectivity of the associated credit risk in the network decreases, and increasingly fewer associated 


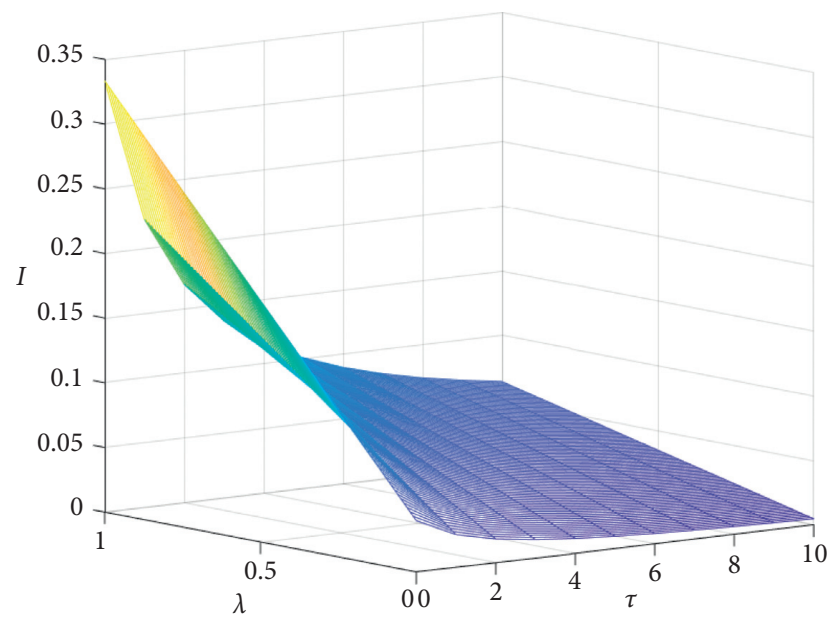

Figure 1: Interaction of the infected entities density $I$, incubatory period $\tau$, and infection probability $\lambda$.

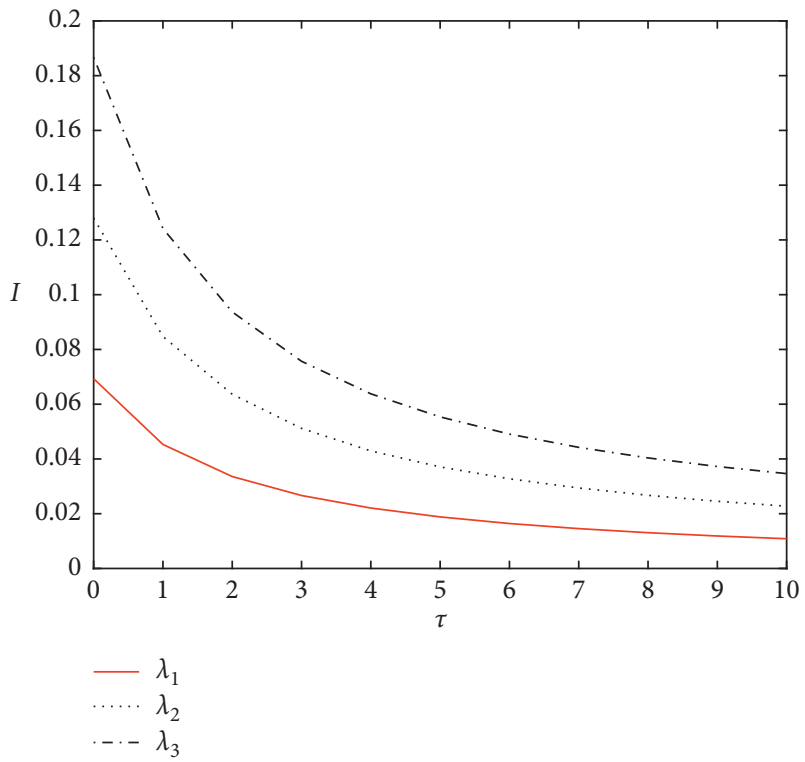

FIGURE 2: Interaction of the infected entities density $I$ and incubatory period $\tau$ considering different infection probabilities $\lambda$.

entities are infected, which is consistent with reality. (ii) When the incubatory period is unchanged, before 1.5 units of time, as the recovery rate increases, the number of infected entities abnormally increases. This observation may be the reason why there is an increase in the number of infected entities who do not receive timely assistance and have weakened resistance, even though the recovery rate increases, which causes the overall density of infected entities to increase in a short time. When the incubatory period is unchanged, at 1.5 units of time, the change in the recovery rate will not affect the overall density of infected entities, and the density of infected entities is constant at this moment. When the incubatory period is unchanged, after 1.5 units of time, the density of infected entities decreases as the recovery rate increases. Therefore, when the incubatory period remains unchanged, the overall change in the density of

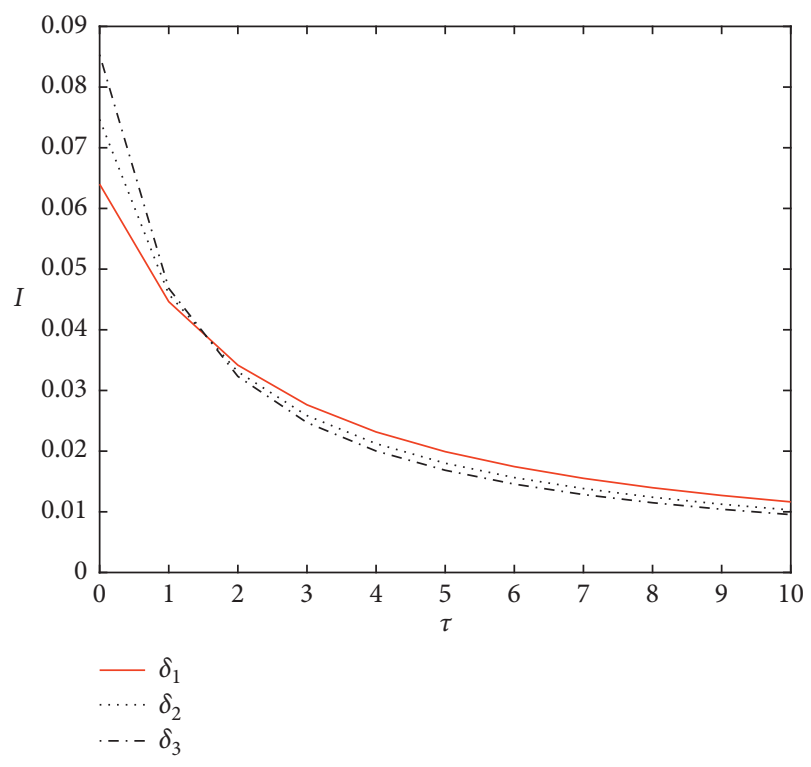

FIGURE 3: Interaction of the infected entities density $I$ and incubatory period $\tau$ considering the different recovery rates $\delta$.

infected entities is in line with the actual situation. It is concluded that the recovery rate of the infected entities and the incubatory period of the latent entities are important factors affecting the density of the infected entities.

Figure 4 shows that the relationship between the density of infected entities $I$ and the incubatory period $\tau$ of latent entities considering different newborn rates of credit entities $M_{1}=0.01, M_{2}=0.05$, and $M_{3}=0.08$ when $\alpha=0.1, w=2$, $M=0.05, m=0.01$, and $\lambda=0.1$. To more clearly explore the contagion of associated credit risk, the new rate here temporarily represents the initial value of the associated entities network, and so the change in the new rate represents the change in the initial value of the network. The following can be seen from Figure 4: (i) when the new rate remains unchanged, as the incubatory period of latent entities increases, the infectivity of the associated credit risk in the network decreases, and increasingly fewer associated entities are infected, which is consistent with reality. (ii) When the incubatory period remains unchanged, the marginal density of infected entities will increase as the new rate increases. That is, the higher the new rate is, the faster the density of infected entities will decrease. (iii) When the incubatory period remains unchanged, the higher the new rate is, the smaller the density of infected entities is. This observation may be due to the greater number of new entities, the increase in the network nodes, and the dilution of the density of infected entities. Therefore, the newborn rate of credit entities and the incubatory period of latent entities are important factors affecting the density of infected entities.

Figure 5 shows that the changing relationship between the density of infected entities $I$ and the incubatory period $\tau$ of latent entities considering different mortality rates of credit entities $m_{1}=0.001, m_{2}=0.01$, and $m_{3}=0.1$ when $\alpha=0.1, w=2, \gamma=0.5, M=0.01$, and $\lambda=0.1$. The following can be found: (i) with the increase in the incubatory period of latent entities, the infectivity of the associated credit risk 


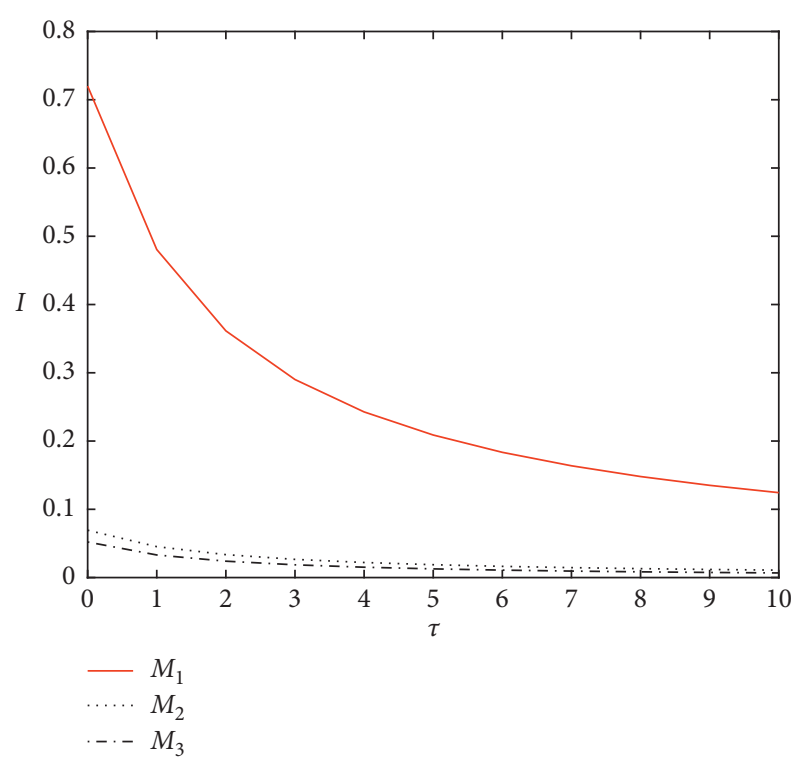

FIgURe 4: Interaction of the infected entities density $I$ and incubatory period $\tau$ considering different newborn rates $M$.

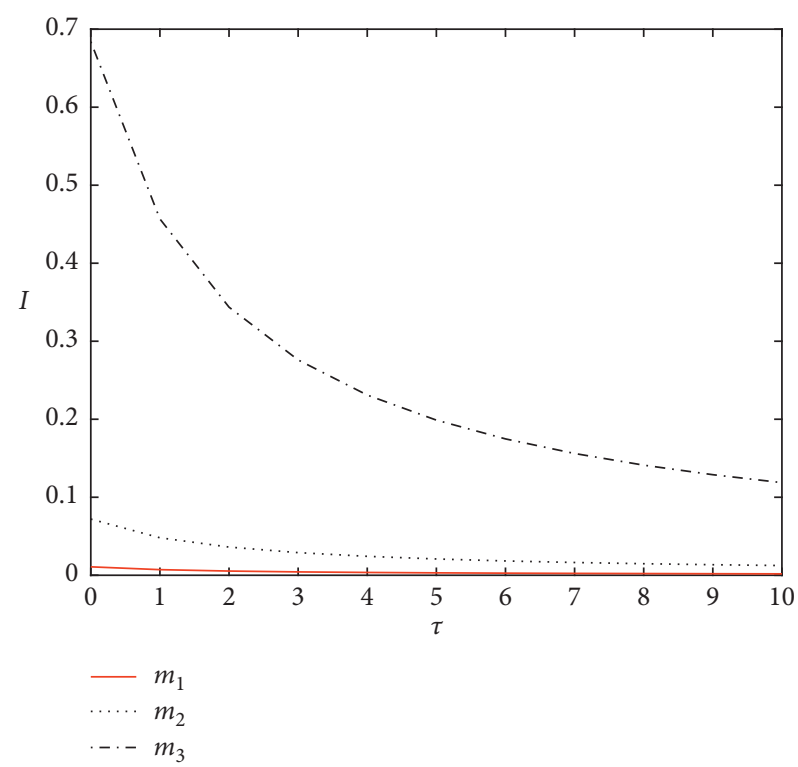

FIGURE 5: Interaction of the infected entities density $I$ and incubatory period $\tau$ considering the mortality rate $m$.

in the network decreases, and increasingly, fewer associated entities are infected. That is, the density of infected entities tends to decrease, which is consistent with reality. (ii) When the incubatory period and other conditions remain unchanged, with the increase in the mortality rate, the density of infected entities will increase. That is, the density of infected entities will increase as the mortality rate increases. This observation may be because most of the dead entities are susceptible entities and new entities. Meanwhile, the infected entities have a lower mortality rate because of their enhanced resistance and self-healing consciousness, and so the density of infected entities will increase. Therefore, the mortality rate of credit entities and the incubatory period of latent entities are important factors affecting the density of infected entities.

\section{Conclusions and Discussion}

With economic globalization and the further development of market economies, the relationships among various credit entities, such as enterprises, banks, insurance companies, and guarantee companies, are becoming increasingly closer and more complex, and the credit transactions among various associated entities are increasingly frequent; therefore, the associated credit risks are widespread. The change in the associated credit risk not only has a chained effect but also has an incubatory period. Once the credit risk breaks out, it will quickly spread to other associated entities, thus harming the network of associated entities, even the financial system and the whole social economy, and even leading to the outbreak of systemic financial risk. Therefore, it is of great theoretical significance and practical value to explore the hidden contagious evolutionary mechanism of associated credit risk and to identify, prevent, and control associated credit risk. However, in practical economic development and business operations, the incubatory period of associated credit risk is not well understood, which leads to missed opportunities for risk control, credit crises, and even the outbreak of global financial crises. The main contribution of this paper is to use the SIS infectious dynamics model and introduce latent entities and the incubatory period to characterize the infectious evolutionary mechanism of associated credit risk in the network of associated entities. Furthermore, in the BA scale-free network, the numerical simulation analysis shows the following: (i) the contagion threshold of associated credit risk is related to the network structure, and it changes in the same direction as the incubatory period of latent entities, the recovery rate of infected entities, and the new rate of credit entities. Meanwhile, the contagion threshold changes in the opposite direction as the infective ability of infected entities. (ii) The longer the incubatory period of the latent entities is, the higher the recovery rate of infected entities is, the higher the mortality rate is, and the smaller the density of the infected entities is. (iii) The higher the probability of infected entities is, the greater the density of infected entities is, the higher the new rate of credit entities is, and the faster the density of infected entities will decrease. The above conclusions are consistent with the crisis phenomena in the real network of associated entities. Furthermore, this paper provides a new perspective for enterprises, banks, guarantee companies, and other credit entities to control associated credit risk. Our future research will apply the data of listed companies in Shanghai and Shenzhen stock exchanges of China to conduct an indepth empirical study on the incubatory period and evolutionary mechanism of the associated credit risk contagion. 


\section{Data Availability}

The simulation data used to support the findings of this study are available from the corresponding author (xukai@cdu.edu.cn) upon request.

\section{Conflicts of Interest}

The authors declare that there are no conflicts of interest.

\section{Acknowledgments}

This work was supported by the National Natural Science Foundation of China (nos. 71701166 and 71871147), Sichuan Social Science Planning Project (no. SC19B004), Soft Science Project of Chengdu Science and Technology Bureau (no. 2016-RK00-00010-ZF), a project supported by Sichuan Landscape and Recreation Research Center (no. JGYQ2018004), and Sichuan County Economic Development Research Center (no. xy2018016).

\section{References}

[1] Z. F. Zhou, Associated Credit Risk and its Contagion EffectImportant Risk Sources in Modern Society, Economy and Market Environment, Science Press, Beijing, China, 2018.

[2] Y. K. Li and Z. F. Zhou, "Contagion delayed effects of associated credit risk based on scale-free network," Systems Engineering, vol. 33, no. 8, pp. 74-79, 2015.

[3] O. De Bandt and P. Hartmann, Systemic Risk: A Survey, Social Science Electronic Publishing, Beijing, China, 2000.

[4] P. Smaga, The Concept of Systemic Risk, Systemic Risk Centre Special Paper, Beijing, China, 2014.

[5] R. M. May, S. A. Levin, and G. Sugihara, "Ecology for bankers," Nature, vol. 451, no. 7181, pp. 893-894, 2008.

[6] G. Luo, Y. W. Zhao, and Y. Wang, "Risk propagation patterns of guarantee network based on the theory of complex network," Journal of University of Chinese Academy of Sciences, vol. 32, no. 6, pp. 836-842, 2015.

[7] P. Xu and X. Yu, "Research on the application of risk contagion model of mutual guarantee financing for SMEs clusters," Accounting Research, vol. 2018, no. 1, pp. 82-88, 2018.

[8] M. Elliott, B. Golub, M. O. Jackson et al., "Financial networks and contagion," American Economic Review, vol. 104, no. 10, pp. 3115-3153, 2014.

[9] E. Fisher, "A biological approach for financial network contagion based on the Susceptible-Infected-Recovered(SIR) model," Anã lisis Econã mico, vol. 21, no. 69, pp. 109-128, 2013.

[10] M. Eboli, "A flow network analysis of direct balance-sheet contagion in financial networks," Journal of Economic Dynamics and Control, vol. 2019, no. 103, pp. 205-233, 2019.

[11] R. Gencay, H. Pang, M. C. Tseng et al., "Contagion in a network of heterogeneous banks," Journal of Banking and Finance, vol. 2020, Article ID 105725, no. 111, p. 16, 2020.

[12] H. Dastkhan and N. S. Gharneh, "Simulation of contagion in the stock markets using cross-shareholding networks: a case from an emerging market," Computational Economics, vol. 53, no. 3, pp. 1071-1101, 2019.

[13] A. Bucci, D. La Torre, D. Liuzzi, and S. Marsiglio, "Financial contagion and economic development: an epidemiological approach," Journal of Economic Behavior \& Organization, vol. 162, pp. 211-228, 2019.
[14] Z. H. Hu and X. H. Li, "Contagion and bailout strategy in complex financial network-SIRS model on the Chinese scale-free financial network," Finance \& Trade Economics, vol. 2017, no. 4, pp. 101-114, 2017.

[15] H. Dastkhan, "Network-based early warning system to predict financial crisis," International Journal of Finance \& Economics, vol. 2019, 2019.

[16] P. Sarlin, "On biologically inspired predictions of the global financial crisis," Neural Computing and Applications, vol. 24, no. 3-4, pp. 663-673, 2014.

[17] P. Giudici, P. Sarlin, and A. Spelta, "The interconnected nature of financial systems: direct and common exposures," Journal of Banking \& Finance, vol. 2020, Artticle ID 105149, no. 112, p. 18, 2020.

[18] N. Apergis, C. Christou, I. Kynigakis et al., "Contagion across US and European financial markets: evidence from the CDS markets," Journal of International Money and Finance, vol. 96, no. 96, pp. 1-12, 2019.

[19] B. Chakrabarty and G. Zhang, "Credit contagion channels: market microstructure evidence from Lehman Brothers'bankruptcy," Financial Management, vol. 41, no. 2, pp. 2320-2343, 2012.

[20] P. Jorion and G. Zhang, "Credit contagion from counterparty risk," The Journal of Finance, vol. 64, no. 5, pp. 2053-2087, 2009.

[21] D. Barro and A. Basso, "Credit contagion in a network of firms with spatial interaction," European Journal of Operational Research, vol. 205, no. 2, pp. 459-468, 2010.

[22] S. Li and X. Sui, "Contagion risk in endogenous financial networks," Chaos, Solitons \& Fractals, vol. 91, pp. 591-597, 2016.

[23] S. Jiang, H. Fan, and M. Xia, "Credit risk contagion based on asymmetric information association," Complexity, Article ID 2929157, vol. 2018, p. 11, 2018.

[24] T. Q. Chen, J. P. Wang, and J. N. Wang, "Research on credit risk contagion mechanism of counterparty based on CDS," Financial Theory \& Practice, vol. 4, pp. 35-38, 2017.

[25] T. Q. Chen, B. Q. Xiao, and H. F. Liu, "Credit risk contagion in an evolving network model integrating spillover effects and behavioral interventions," Complexity, vol. 2018, Article ID 1843792,, p. 16, 2018.

[26] L. Li and Z. F. Zhou, "Credit contagion mechanism for interrelated guarantee," Systems Engineering, vol. 1, pp. 55-60, 2015.

[27] H. J. Yang and H. M. Hu, "Risk contagion of Chinese interbank markets based on core-periphery network," Journal of Management Sciences in China, vol. 20, no. 10, pp. 44-56, 2017.

[28] L. Wu, Y. M. Zhuang, and L. Zhang, "An analysis of credit risk diffusion in inter-bank market," Journal of Southeast University(Philosophy and Social Science), vol. 19, no. 6, pp. 74-79, 2017.

[29] L. Wang, S. Li, T. Chen et al., "Investor behavior, information disclosure strategy and counterparty credit risk contagion," Chaos, Solitons \& Fractals, vol. 119, pp. 37-49, 2019.

[30] S. Jiang and H. Fan, "Credit risk contagion coupling with sentiment contagion," Physica A: Statistical Mechanics and Its Applications, vol. 512, pp. 186-202, 2018.

[31] C. Giusy, G. Gianfranco, and S. Marco, "Climate change and credit risk," Journal of Cleaner Production, vol. 2020, Article ID 121634, no. 266, p. 18, 2020.

[32] V. De Bruyckere, M. Gerhardt, G. Schepens, and R. Vander Vennet, "Bank/sovereign risk spillovers in the European debt crisis," Journal of Banking \& Finance, vol. 37, no. 12, pp. 4793-4809, 2013. 
[33] J. Breckenfelder and B. Schwaab, "Bank to Sovereign risk spillovers across borders: evidence from the ECB's comprehensive assessment," Journal of Empirical Finance, vol. 49, pp. 247-262, 2018.

[34] M. Greenwood-Nimmo, J. Huang, V. H. Nguyen et al., "Financial sector bailouts, sovereign bailouts, and the transfer of credit risk," Journal of Financial Markets, vol. 42, pp. 121-142, 2019.

[35] S. Srivastava, H. Lin, I. M. Premachandra, and H. Roberts, "Global risk spillover and the predictability of sovereign CDS spread: international evidence," International Review of Economics \& Finance, vol. 41, pp. 371-390, 2016.

[36] G. Bostanci and K. Yilmaz, "How connected is the global sovereign credit risk network," Journal of Banking and Finance, vol. 2020, no. 113, Article ID 105761, 2020.

[37] K. Xu, Z. F. Zhou, and Q. Qian, "The impact of risk information and behavioral response on associated credit risk contagion," Technology Economics, vol. 37, no. 8, pp. 116-122, 2018.

[38] Q. Qian and Z. F. Zhou, "Research on the associated credit risk within the immunization," Operations Research and Management Science, vol. 2018, no. 1, pp. 132-137, 2018.

[39] L. Li and Z. F. Zhou, "Dynamic infection mechanism research for credit risk within business group," Management Review, vol. 2015, no. 1, pp. 48-56, 2015.

[40] Q. Qian, Y. Yang, J. Gu, and H. R. Feng, "Information authenticity, spreading willingness and credit risk contagion-A dual-layer network perspective," Physica A: Statistical Mechanics and Its Applications, vol. 536, Article ID 22519, 2019.

[41] H. F. Zhang, J. Xie, H. Chen et al., "Impact of asymptomatic infection on coupled disease-behavior dynamics in complex networks,” EPL, vol. 114, no. 3, p. 6, 2016.

[42] Q. Redouane and A. Hammoumi, "A stochastic delay model of HIV pathogenesis with reactivation of latent reservoirs," Chaos Solitons \& Fractals, vol. 2020, no. 132, Article ID 109594, p. 11, 2020.

[43] T. Shi, T. Long, Y. Pan et al., "Effects of asymptomatic infection on the dynamical interplay between behavior and disease transmission in multiplex networks," Physica A-Statistical Mechanics and Its Applications, vol. 2019, Article ID 121030, no. 536, p. 16, 2019.

[44] R. X. Zhang, Z. Jin, and S. P. Li, "Epidemic spreading with time delay on complex networks," Acta Mathematicae Applicatae Sinica, English Series, vol. 32, no. 2, pp. 319-326, 2016.

[45] C. Y. Xia, Z. Wang, J. Sanz, S. Meloni, and Y. Moreno, "Effects of delayed recovery and nonuniform transmission on the spreading of diseases in complex networks," Physica A: Statistical Mechanics and Its Applications, vol. 392, no. 7, pp. 1577-1585, 2013.

[46] F. Viviana and M. Lucia, "A nonlinear dynamic model for credit risk contagion," Mathematics and Computers in Simulation, vol. 174, no. 8, pp. 45-58, 2020.

[47] Y. K. Li and Z. F. Zhou, "Contagion delayed effects of associated credit risk based on scale-free network," Journal of Systems Engineering, vol. 30, no. 5, pp. 575-583, 2015.

[48] Y. K. Li and Z. F. Zhou, "Contagion and simulation of interfirm associated credit risk based on incomplete immunization," Chinese Journal of Management Science, vol. 25, no. 1, pp. 57-64, 2017.

[49] R. Pastor-Satorras and A. Vespignani, "Epidemic dynamics in finite size scale-free networks," Physical Review E, vol. 65, no. 3, Article ID 035108, 2002.
[50] R. Pastor-Satorras and A. Vespignani, "Epidemic spreading in scale-free networks," Physical Review Letters, vol. 86, no. 14, Article ID 3200, 2001.

[51] W. O. Kermack and A. G. Mckendrick, "Contributions to the mathematical theory of epidemics. II. the problem of endemicity," Proceedings of The Royal Society A: Mathematical, Physical and Engineering Sciences, vol. 138, no. 834, pp. 55-83, 1932.

[52] K. Xu, Z. F. Zhou, and Q. Qian, "Impact of information bidirectional dissemination and individual response on associated credit risk contagion," Systems Engineering, vol. 38, no. 2, pp. 11-19, 2020. 